\title{
Wireless Control and Monitoring of Industrial Processes by AVR and LabVIEW*
}

\author{
Tamara Z. Fadhil \\ Department of Informatics Systems Manage \\ Businesses Informatics College \\ University of Information Technology and Communications \\ Baghdad, Iraq \\ tamara.zuhair@uoitc.edu.iq
}

\begin{abstract}
This paper presents expansion, enhancement, design and implementation of wireless SCADA systems to control a variety of industrial processes from a master location which include authorizing vast monitoring for pipelines, natural gas, petroleum fields, water and transportation systems, chemical plants, utilities, oil refineries, power generation, electrical grid and an immense assortment of industrialization functionality. The control of environmental temperature, oil pressure, gases concentration, fuel tank level in power industrial plant had been collected and analyzed with a high power capacity 1 MW, based on Master terminal unit (MTU) design for data acquisition from Remote Terminal Unit (RTU) sensors and wireless data transfer using $2.4 \mathrm{GHz}$ RF module, makes sensors data collection come true.
\end{abstract}

Index Terms - SCADA Systems, Remote Monitoring, LabVIEW, AVR.

\footnotetext{
* The paper has been presented in the 4th International Scientific
} Conference of University of Human Development April, 2017.

\section{INTRODUCTION}

Wireless communication is ultimately one of the most vibrant zones in the scope of communication today. However, it has been a topic of research since the 1960s. The last decade has seen a burst of projects and activities in this area due to a concourse of varied factors. Specifically, a tremendous increase in demands of connectivity, higher data rates, synchronous and asynchronous data transmission and rapid introduction of new services and technologies [1]. Examples would include development industries related to wireless communications in the electrical power system, working on improving reliability and proficiency of power generation by adjusting and supervising networks within the electrical power scheme.

\section{LITERATURE REVIEW}

Acquiring multiple data sets using different sensors' network topologies, the information may be analog or discrete in nature for the domain. Operation at high speed may employ a multichannel data acquisition system. Processing the data with the aid of a data processing algorithm and computing equipment, as well as presenting the data for the employer is the primary necessity of any industrial automation system. Several methods of data acquisition, data logging, supervisory control and modem development had been discussed in the field. Also, the distinct control requirement and element control scheme for the boiler unit had been discussed [2].

The surveillance and construction of a microcontroller PIC16 and LabVIEW-based SCADA system had been designed for control and enhancement of the performance by acquiring and manipulating physical parameters such as temperature, soil moisture, humidity and intensity of light in the greenhouse system on a real- time basis. This 
study uses software such as LabVIEW, together with a low-cost microcontroller PIC16 based data acquisition hardware, as DAQ card had been described in [3].

Consideration of the communication and security components are popular among these energy subsystems. Supervisory Control and Data Acquisition (SCADA) is the essential subsystem of a smart grid. A second key component of a smart grid is to recognize all connections to SCADA networks and how well these connections are secured, as well as those that are highly available in wireless networks. The wireless technologies include WiMAX, WLAN, WAN, all versions of cellular technologies and wireless sensor protocols. The comprehensive security setting is the key component as privacy is still the main stumbling block to a realization of smart grid technology [4].

This paper is submitting challenges to improve a Data Acquisition Card (DAQ) and data logging system that supervise influential parameters of the electrical power system, i.e. measure voltage and temperature, current in three-phase systems. If the temperature, fuel levels or oil pressure go over of bounds then subsequent control action is applied to maintain the normal level .The advantages of wireless sensors network which is suitable for deployment in the electrical power system.

\section{SUPERVISORY CONTROL AND DATA ACQUISITION SYSTEMS (SCADA)}

The acronym SCADA symbolize Supervisory Control and Data Acquisition. Factually, the essential objective of SCADA is to control, monitor, and alarm plant or regional implementing systems from a centric location. Although override control is possible, it is infrequently employed; nevertheless control set factors are completely regularly altered by SCADA [5]. The pioneer attraction of SCADA to a municipality is the capability to significantly diminish operating action costs, and improve plant or regional system performance and reliability. Data gathering in a plant no longer requires staff to spend time roaming all around the site, and consequently the frequency of field site investigation required in a regional system can be decreased [6]

The term SCADA generally refers to a main system that monitors and controls a complete location. The bulk of the location control is actually executed automatically by a Remote Terminal Unit (RTU) or by a Programmable Logic Controller (PLC) [7, 8]. The PLC is a processing computer, real-time system for several inputs and output configuration, extensive temperature gauges, inviolability to electrical noise, impedance to vibration.

Server control functions are almost always limited to basic site over-ride or supervisory level capability. Data acquisition begins at the RTU or PLC level, involves meter senses and tool statuses that are connected to the SCADA as fundamentally. Data is already compiled and arranged in such a method that a supervise chamber operator employing the SCADA enable to make a suitable supervisory decisions that may be desired in order to over-ride normal RTU (PLC) controls.

SCADA systems are used not only in industrial processes: e.g. distribution, steel making, and power 856 generation (conventional and nuclear), but also for natural gas conveyance and allocation systems. SCADA systems used to run on DOS, VMS and UNIX; in recent years all SCADA dealers have moved to NT and some as well to Linux [9].

\section{PROPOSED SYSTEM}

In this section we will discuss the building of an Industrial power Management system from four points: A. Hardware Architecture of $M T U$

A performance and powerful control system has been implemented with a high power capacity $1 \mathrm{MW}$, which can optimize power usage, quality and reliability in an industrial environment, which is an important aspect of improving costs critical to profitability.

Master terminal unit (MTU) design for data acquisition from RTU sensors in an industrial plant, that allows for operators to have several beneficial features, and it makes their work flexible and easy. It gives numerous options to deal with the alarms. It provides the users with fault events, graphical displays and opportunity to change texts.

The historical databases are used for analyzing events and making reports to provide clear views about the operation and business.

Reading voltages, emerging pregnancy currents, timing delivery of extra capacity and control the temperature and the oil pressure inside the engine, each phase called voltage indicator for the three phases. There are also controls one of them to connect the feed battery for generating the second key to initially operating advances starting motor is called the master key, which connect beyond the ability of consumer. Also, there are three identical keys for each phase order is controlled on each phase are isolated from each other. The interface program is structured timer to determine the runtime and extinguish generated and connected load electricity to nourishing a time of their choice. Figure. 1 shows the simulation of the proposed system that interfaces with PLC which permits the operators to monitor the power plant with a personal computer. Figure 2 shows the Master terminal unit (MTU) hardware architecture.

Table I presents the main device data used in this system. 


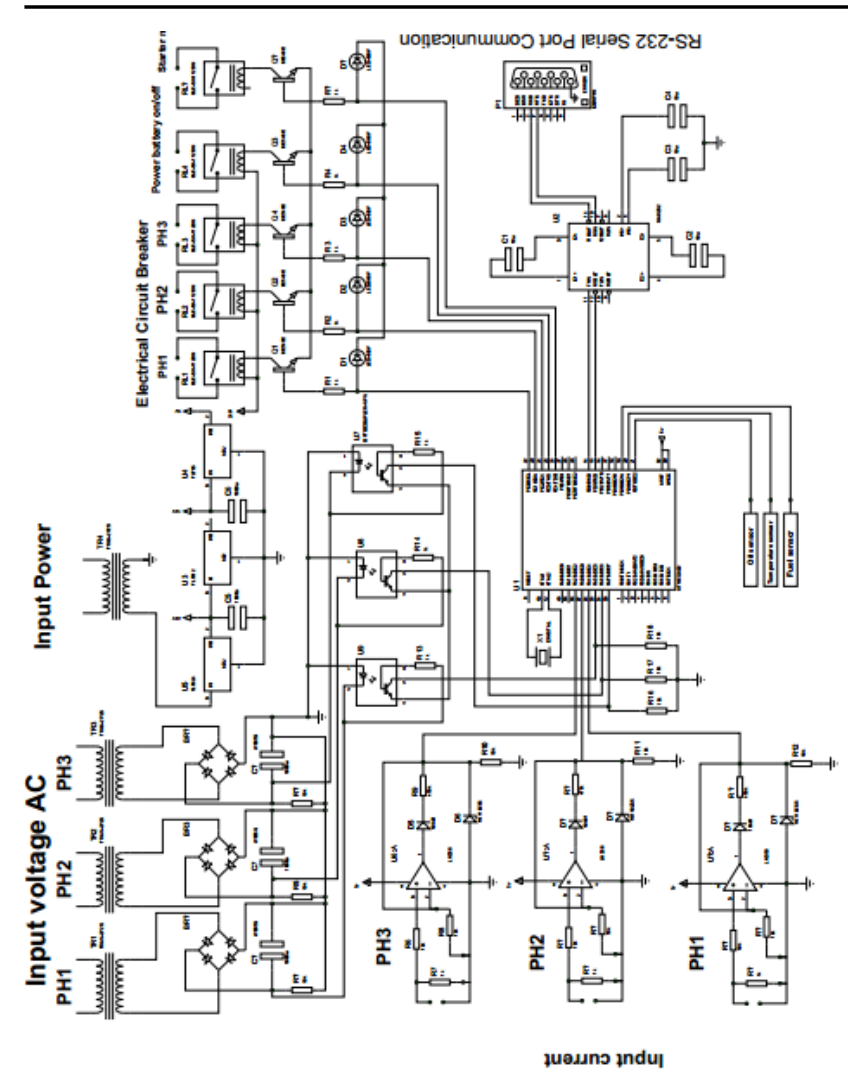

Fig.1 Simulation of proposed system

TABLE I

DATA OF PROTOTYPING HARDWARE COMPONENTS

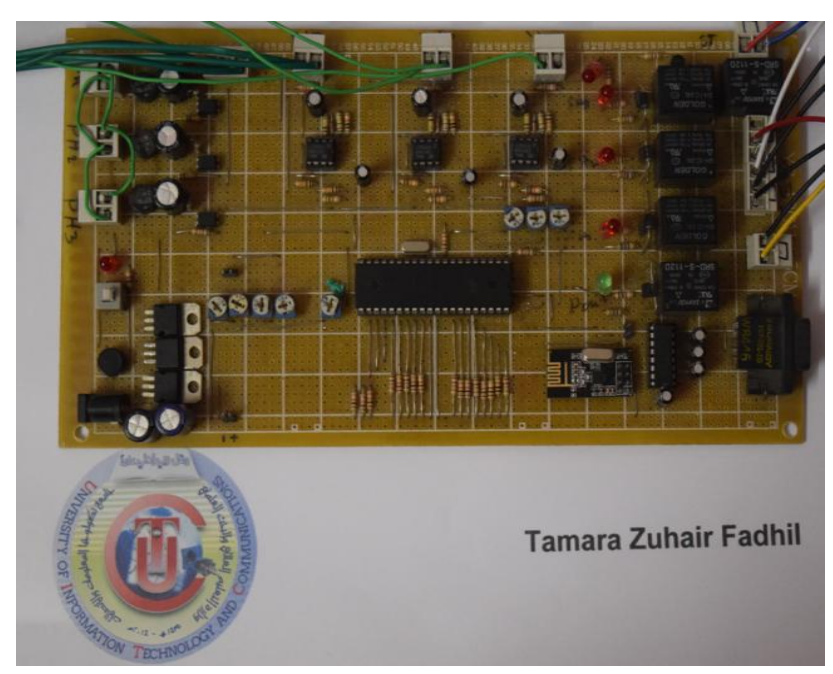

Fig.2 Prototyping hardware as (MTU)

\section{- $\quad$ Condition monitoring}

Condition Monitoring is the operation of surveilling all electrical, mechanical and peripheral limitations that could have any unfavorable effect or could influence the system in a damaging way, resulting in unnecessary downtime or high cost. Various condition monitoring sensors in the system, including fuel level, two pole VDO oil pressure sender sensor, and VDO temperature sensor will supply input signals to the 857 regulator. Then, it will subsequently use this realization to overcome performance problems like production jams or mechanical insufficiency authorize the premise of reliability and concentrated maintenance for the integral system.

\section{B. Virtual instrumentation design using LabVIEW}

LabVIEW is a graphical programming language can that be used to solve various problems. Having vigorous problem-solving skills and robust background in mathematics and engineering technology basics are essential to produce solutions in LabVIEW. LabVIEW stands for Laboratory Virtual Instruments (VIs) Engineering Workbench (LabVIEW). VIs include three major elements - the front panel, the icon/connector pane and the block diagram. So as to utilize a VIs as a sub-VIs in the block diagram of another VIs, it is important that it have a connector and an icon. The LabVIEW windows are the front panel (containing controls and indicators) and block diagram (containing terminals, connections and graphical code). The front panel is the user interface of the virtual instrument. The code is built using a graphical illustration, which is importance to surveil the front panel target. The block diagram window implicates this graphical source code as shown in figure $r$.

Subsequent software and drivers had been installed, the next step is to physically connect and then set up our proposed hardware. With LabVIEW, we have simulates the wireless control block diagram, receiving data and

\begin{tabular}{|c|c|}
\hline Electronics Components & Value \\
\hline Zener Diode & $5 \mathrm{~V}$ \\
\hline Microcontroller & ATMEGA32 \\
\hline Transistor & $1000 \mu \mathrm{f} / 25 \mathrm{v}$ \\
\hline Capacitor & $12 . \mathrm{MHZ}$ \\
\hline Crystal Oscillator & $1 \mathrm{~m} 358$ \\
\hline Operational Amplifier & Max.232 \\
\hline IC & $1 \mathrm{~K} \Omega / 10 \mathrm{~K} \Omega / 470 \mathrm{~K} \Omega$ \\
\hline Resistors & $24 \mathrm{~V}$ \\
\hline Optocoupler & $5 \mathrm{v} / 12 \mathrm{v} / 24 \mathrm{v}$ \\
\hline Relay & $220 \mathrm{v}$ to $12 \mathrm{v}$ \\
\hline Voltage Regulator & \\
\hline Transformer & $80 \mathrm{psi}$ \\
\hline Bridge diode & SSS/SSL Diesel \\
\hline diode & Measuring range: \\
\hline VDO sensor & $0-1.0 \mathrm{Mpa}$ \\
\hline Fuel Level Sensor & NRF24L01 \\
\hline Oil Pressure Gauge Sensor & \\
\hline Wireless & isplays the
\end{tabular}

displayed to the user in the form of curves. It displays the values and issue reports that explain the industrial process, alerts for high temperatures or lack level of fuel or liquid. 


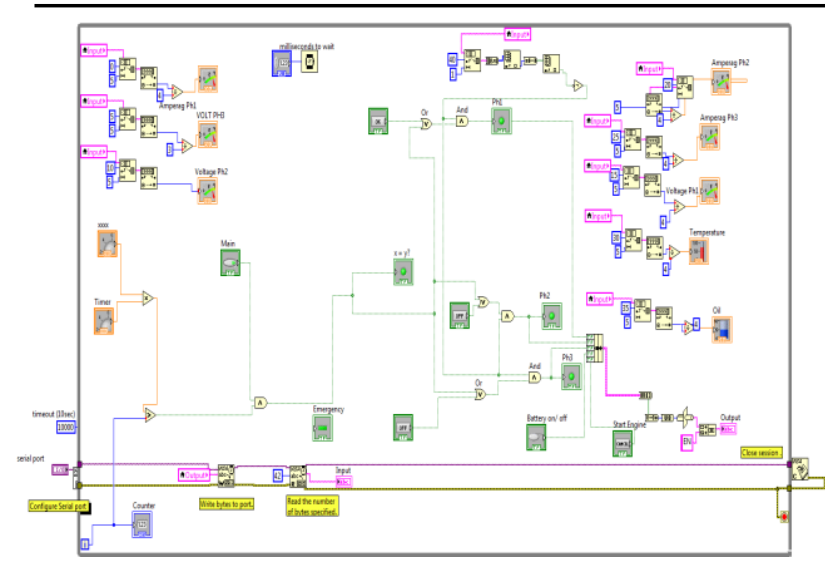

Fig. ${ }^{r}$ LabVIEW simulates of wireless control In order to quickly realize the front panel and block diagram

\section{SCADA Client/Server Configuration}

There are two main parts of any SCADA software, the server and the client:

1) SCADA-PC as Server

Human machine interface (HMI) is responsible for core SCADA functions, such as data communication with the control hardware (either directly or via OPC server), graphics creation, alarm generation, data storage, security, reports generation ... etc. as shown in figure 4 .

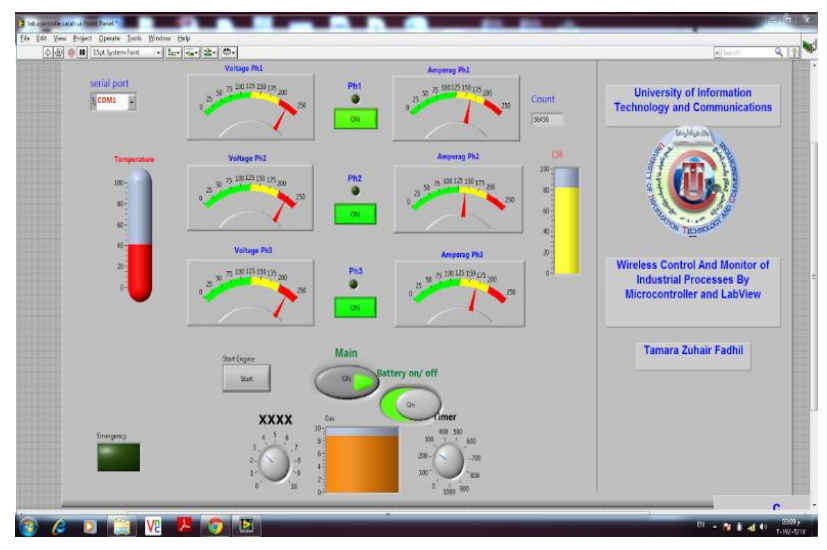

Fig. \& Supervisory computer system HMI normal display

\section{2) SCADA - PC as Client}

Exhibits data to the employee, equips for surveillance inputs and pass operator commands to the SCADA server in a diversity of formats, like graphics screens, schematics, pull-down menus, windows, trends and reports display ... etc. as shown in figure 5.

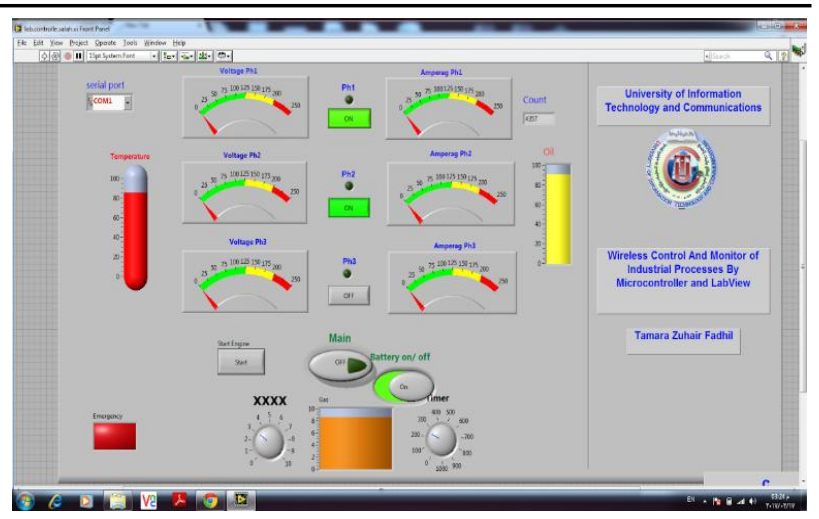

Fig. ${ }^{\circ} \mathrm{HMI}$ real time emergency monitoring by client

\section{Transceiver and Data Collecting with remote terminal unit (RTU)}

Often there are problems associated with the need to obtain data from sensors located at a significant distance from the control device. A simple solution: stretch a couple of tens of meters of wire. However, this is not always possible. Long wires are prone to interference. In addition, the supply of power over long wires is often impossible due to the high resistance of the conductor. Therefore, the power supply still must be placed in the immediate vicinity of the sensor. Do not forget the need to hide the wires for a minimal aesthetic appeal as well. All of this pushes us to the idea of dispensing completely without wires, namely, to build a system for transmitting data over a radio channel. There is also an inexpensive solution for wireless communication: a radio module based on the NRF24 chip. Connection and work with one of the varieties of such a chip and will be discussed in the article.

The NRF24L01 is a transceiver designed for ultralow power wireless purpose which is a single chip device operates under $2.4 \mathrm{GHz}$ with an embedded baseband protocol impulse. It is configured and operated out of a Serial peripheral Interface (SPI.) The register sketch includes all the registers in the nRF24L01 that supports data rate up to $2 \mathrm{Mbps}$. It consists of 4 modes of operation namely RX mode, TX mode, standby mode and Power Down mode [10].

Controlling and monitoring of wireless data transfer using network of $2.4 \mathrm{GHz}$ RF Modules as shown in figure 6 .

Figure 7 shows NRF24L01's work flowchart in proposed system. 


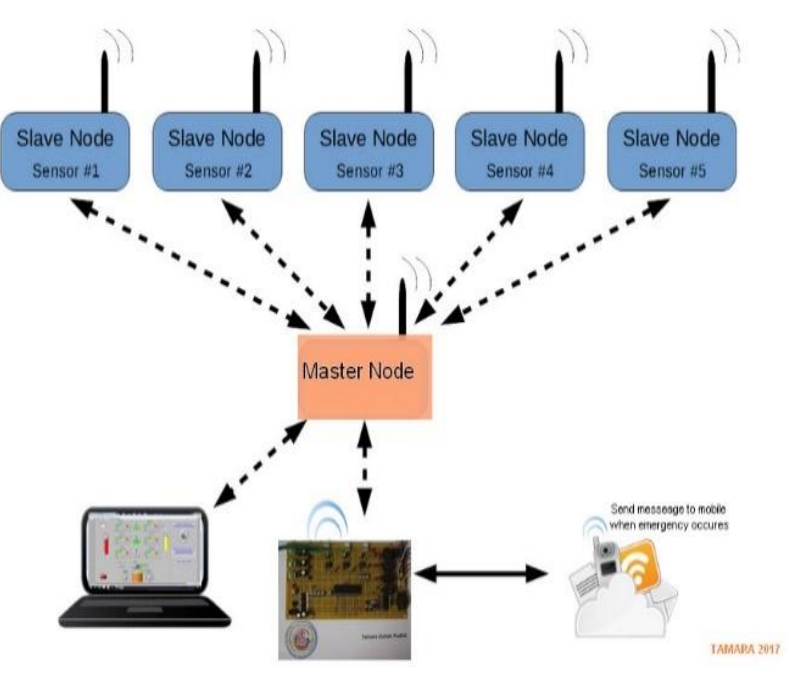

Fig.6 Proposed system network

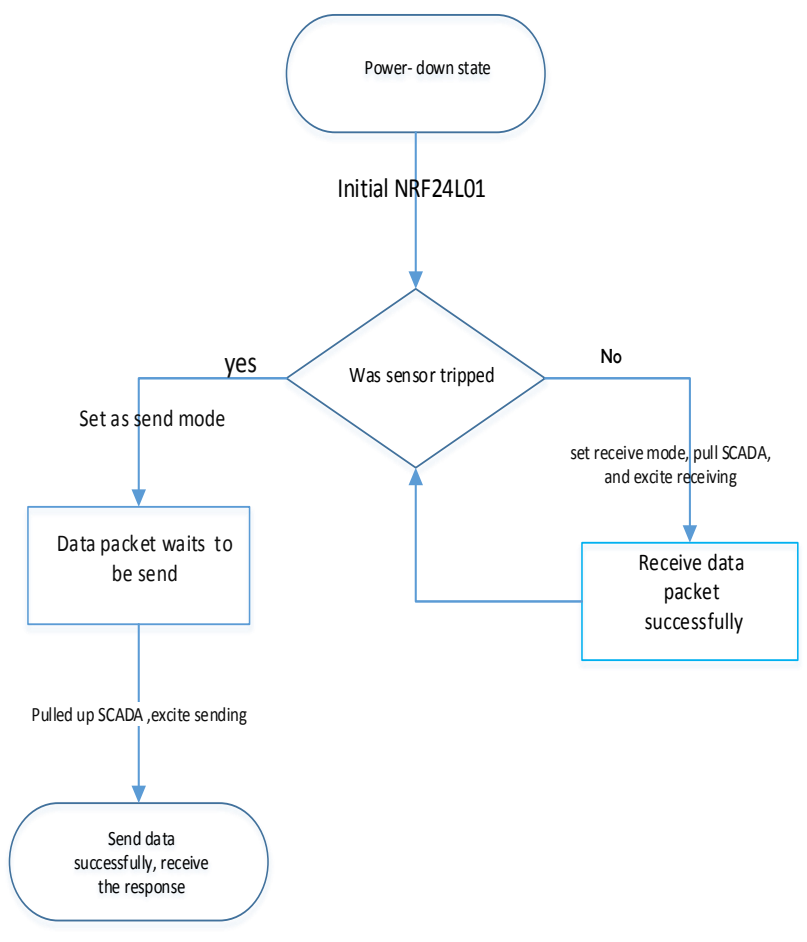

Fig.7 NRF24L01's work flowchart

The FRDM-KL25Z is an ultra-low-cost development platform for Kinetis L Series KL1x (KL14/15) and KL2X (KL24/25) MCUs built on 32-bit ARM Cortex-M0+core processor up to $48 \mathrm{MHz}$ operation, with extension board selections and a built-in debug interface for flash programming and run-control [11].

Five nodes of remote terminal unit (RTU) will receive measurements through sensors and then forward measurements to a central (master) node with LabVIEW as shown in figure 8 .

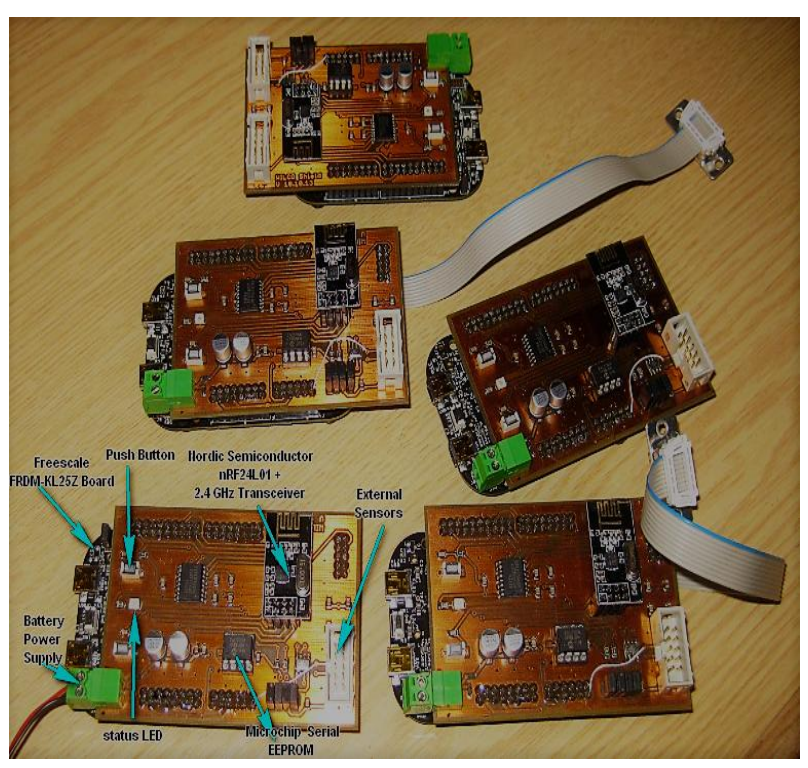

Fig. 8 Network nodes of low cost wireless transceiver with the FRDM board as RTU

\section{RESULTS AND DISCUSSIONS}

- $\quad$ Sending data (un guided) using NRF

Finally, it sends a pulse with the CE pin (Chip Enable activates TX or RX mode) of at least $10 \mu \mathrm{s}$ to initiate the sending of the data over the space. As shown in figure 9.

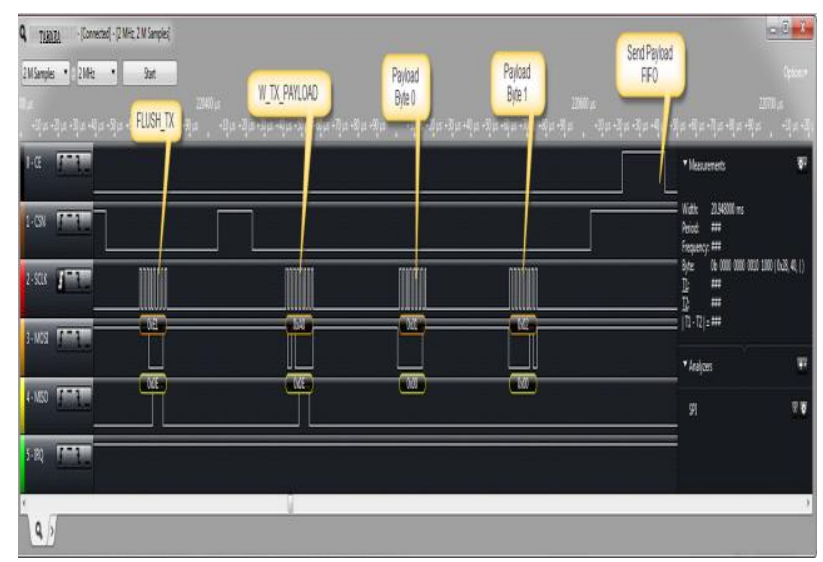

Fig.9 TX_pulse with the CE pin with Flush register

- Setting the interrupt mask bits in the CONFIG register and screening the interrupt flag and resetting the Status flags as shown in figure 10. 


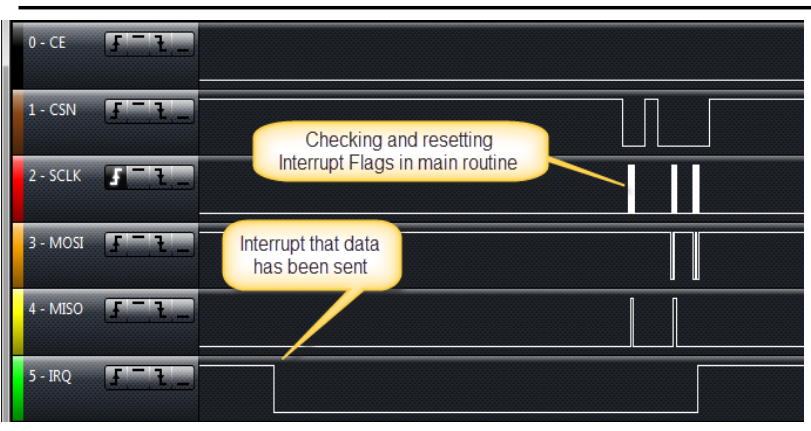

Fig.10 Interrupt flag signal after data has been sent

- Receiving data using network nodes of NRF If data had been sent and acknowledge signal would have received, the $0 \times 20$ bit is set as shown in figure 11 .

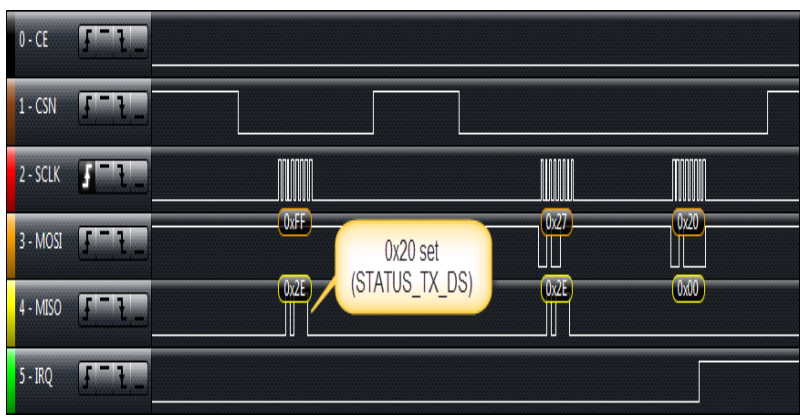

Fig.11 Interrupt with complete data receive

- Variable data had been collected from different sensors such as pressure, temperature, gas concertation from an industry field.

Figure 12 shows the relation between the resistance values of VDO sensor with oil pressure measured from the field. In the system, five volts reference voltage was supplied to the VDO pressure sensor. The pressure sensor measured values from zero to ten bar as you can see in the graph. Resistance was increased dramatically from (10 to $184 \mathrm{ohm}$ ) as the pressure was amplified from zero to ten.

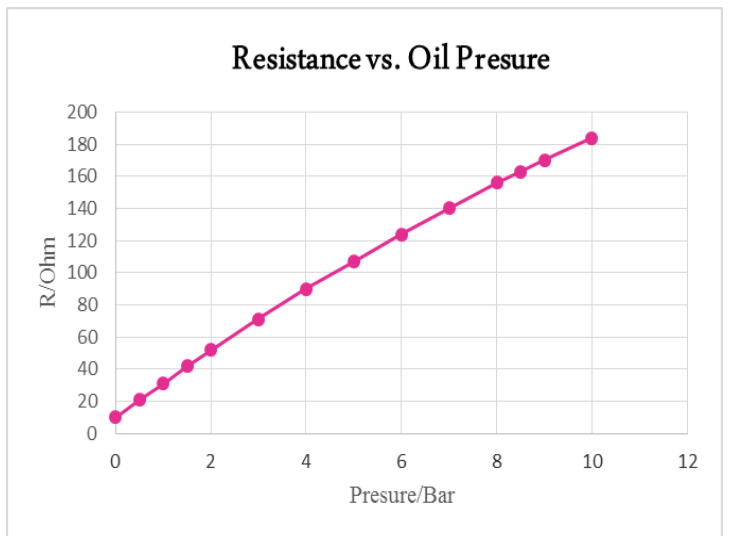

Fig. 12 Pressure sensor reading with resistance value variation

For the temperature measurement, a VDO temperature sensor with a negative coefficient was used to obtain the readings from the field. Also, five volts reference supply was used. The line graph in figure 13 illustrates the temperature readings variation with the resistance values change. The temperature was increased from $\left(0\right.$ to $\left.160^{\circ} \mathrm{C}\right)$ during the experiment. Conversely, the resistance value was dropped from 3240 to $160 \mathrm{ohms}$.

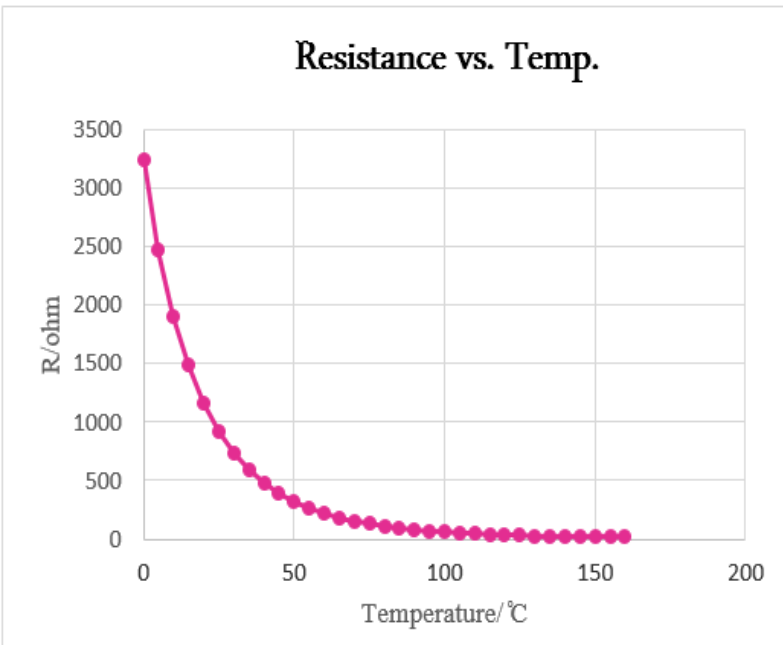

Fig. 13 Temperature sensor reading with resistance value variation

The third sensor is a gas sensor (MQ5). MQ5 is a sensor used for gas infiltration detection such as $\mathrm{H} 2$, LPG, CH4, CO and Alcohol. In the research, three readings data $(\mathrm{H} 2, \mathrm{CO}$ and cleared air) were collected to demonstrate the system working. Figure 14 shows the concentration of $\mathrm{H} 2, \mathrm{CO}$ and cleared air in the field. For all $\mathrm{H} 2, \mathrm{CO}$ and cleared air, the concentration was between 200 and 10000 PPM (part per million).

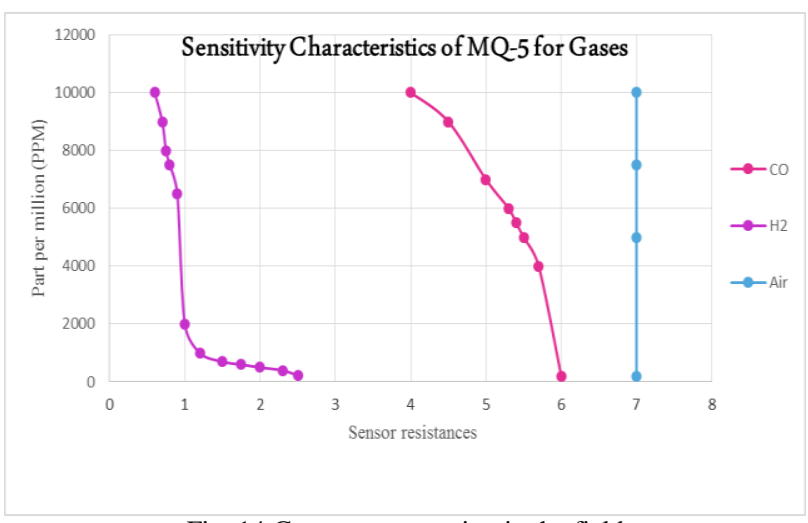

Fig. 14 Gases concentration in the field

Finally, fuel tank level sensor had been used. Figure 15 shows the relation between the fuel level measured with the sensor's resistance. The four sensors readings show the system works correctly by monitoring sensors data founded in an industry field. 


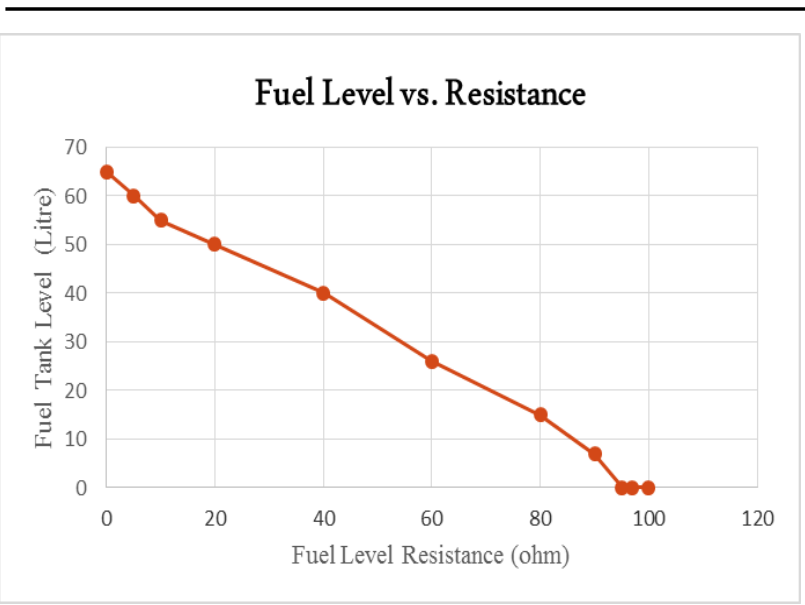

Fig. 15 fuel tank level sensor measurement

\section{CONCLUSIONS}

The design of Wireless Control and Monitoring of Industrial Processes are described in this paper. It approves that:

- The use of active low pass filters will reduce noise in slave node sensor measurements. The filter portion is intended to attenuate high frequency noise from the sensor signal to boost measurement accuracy and ensure maintain max resolution. The low power dual operational amplifiers part scales the signal up to the full scope of the ADC to maintain getting max resolution.

- Using NRF 24 wireless transceiver current consuming is extremely low, only $10.5 \mathrm{~mA}$ at an output power of $-5 \mathrm{dBm}$ and $18 \mathrm{~mA}$ in receive process and built-in power down process creates power saving facilely adaptable.

- Remote monitoring of VDO, fuel level, oil pressure and gases concentration sensors uses a wireless transceiver with the FRDM board as the slave node and implemented prototyping hardware as the master node.

- Virtual Instrumentation VI (highly productive software) had been used for improving system productivity, safety, reliability, optimization and stability. It extends to computerized systems for controlling processes based on data collected and processed by a computerized instrumentation system. The control panel function of the tool is doubled in the computer interface. Dominant from master PC, an external criterion hardware devices and display, test or total data gathered using the extrinsic device on the instrument such as panels on PC display.

- Reduces cost and preserves investment with real-time control needed for device monitoring and test systems.

- The security of the proposed system's ability to resist system difficulties related to mistakes or unlicensed activities without loss services, compromises of human safety and hinders production.

\section{REFERENCES}

[1] N Yee, J-P. Linnartz and G Fettweis, Multicarrier CDMA in Indoor Wireless Radio Networks, Proc of IEEE PIMRC '93, Yokohama, Japan, Sept 1993, pp 109-13.

[2] Subhransu Padhee, Yaduvir Singh, "Data Logging and Supervisory Control of Process Using LabVIEW," Students' Technology Symposium, IEEE, 2011.

[3] Nishantkumar D. Gajipara, Prashant L. Ahire, "Design of SCADA for Real Time System with LabVIEW and Microcontroller," IJIRAE, 2014 pp 85-90.

[4] Anthony R. Metke, Randy L. Ek1, "Security Technology for Smart Grid Networks," IEEE Transaction on Smart Grid, Vol. 1, No. 1, June 2010.

[5] Niladri Kumar Mitra, Principles of Artificial Lift , 1st ed, Allied Publishers Pvt. Ltd., 2012, pp. 361-363.

[6] S\&S IT Innovations Technology SDN BHD, "Automation and Embedded Solutions," 2015.

[7] Yasar Birbir, H.Selcuk Nogay, "Design and Implementation of PLC- Based Monitoring Control System for Three-Phase Induction Motors Fed by PWM Inverter,' international journal of systems applications, engineering development, 2008.

[8] Ali Gulabi, "Development of an Embedded SCADA System with PLC and Java Application for Synchronous Operation of Standard Servo Drives,"Master thesis, Faculty of Engineering and Computer Science of the University of Applied Sciences Hamburg, 2007.

[9] A. Zanasi, Data Mining VIII: Data, Text and Web Mining and Their Business Applications, WIT Press, 2007.

[10] Nilmini Wickramasinghe, Handbook of Research on Healthcare Administration and Management, Epworth HealthCare, Australia and Deakin University, 2017.

[11] http://www.nxp.com/products/power-management/engine-and-dcmotor-control/powertrain-and-engine-control/freedomdevelopment-platform-for-kinetis-k114-k115-k124-k125mcus:FRDM-KL25Z. 